Pacific

Journal of

Mathematics

\title{
INDUCTIVE ALGEBRAS FOR TREES
}

\author{
Giovanni Stegel
}




\title{
INDUCTIVE ALGEBRAS FOR TREES
}

\author{
Giovanni Stegel
}

Let $G$ be a locally compact group and $\pi: G \rightarrow \mathcal{U}(\mathcal{H})$ a unitary representation of $G$. A commutative subalgebra of $\mathcal{B H}$ is called $\pi$-inductive when it is stable through conjugation by every operator in the range of $\pi$. This concept generalizes Mackey's definition of a system of imprimitivity for $\pi$; it is expected that studying inductive algebras will lead to progress in the classification of realizations of representations on function spaces. In this paper we take as $G$ the automorphism group of a locally finite homogeneous tree; we consider the principal spherical representations of $G$, which act on a Hilbert space of functions on the boundary of the tree, and classify the maximal inductive algebras of such representations. We prove that, in most cases, there exist exactly two such algebras.

\section{Introduction. Inductive algebras}

Let $G$ be a separable locally compact group and $\pi: G \rightarrow \mathcal{B}(\mathcal{H})$ a unitary representation of $G$ on a Hilbert space $\mathcal{H}$. A classical tool for studying the realizations of $\pi$ on function spaces is the concept of a system of imprimitivity for $\pi$. In Mackey's formulation a self-adjoint, weakly closed commutative subalgebra $\mathcal{A}$ of $\mathcal{B}(\mathcal{H})$ is called a system of imprimitivity for $\pi$ when $\pi\left(g^{-1}\right) \mathcal{A} \pi(g)=\mathcal{A}$ for every $g \in G$. Given such an algebra, by Mackey's imprimitivity theorem $[8$, Theorem 3.10] there exist a $G$-space $X$, a measure $\mu$ on $X$, a cocycle $A$ and a Hilbert space $\widetilde{\mathcal{H}}$ such that:

- $\pi$ is equivalent to the representation $\pi_{X}$ on $L^{2}(X, d \mu, \widetilde{\mathcal{H}})$ defined in the following way:

$$
\left(\pi_{X}(g) f\right)(x)=A(g, x)\left(\frac{d \mu\left(g^{-1} x\right)}{d \mu(x)}\right)^{1 / 2} f\left(g^{-1} x\right) .
$$

- Through the equivalence, $\mathcal{A}$ corresponds to $L^{\infty}(X, d \mu)$, regarded as an algebra of multiplication operators on $L^{2}(X, d \mu, \widetilde{\mathcal{H}})$.

More generally, given $\pi: G \rightarrow \mathcal{B}(\mathcal{H})$ as above, define a (not necessarily self-adjoint) subalgebra of $\mathcal{B}(\mathcal{H})$ to be $\pi$-inductive algebras when it is 
commutative and stable through conjugation by $\pi$. An operator $T \in \mathcal{B}(\mathcal{H})$ belongs to some $\pi$-inductive algebra if and only if

$$
\left[T, \pi(g) T \pi\left(g^{-1}\right)\right]=0 \quad \text { for all } g \in G .
$$

If $\pi$ can be realized as acting on a space of functions on $X$ (not necessarily an $L^{2}$-space) it often happens that there is an associated $\pi$-inductive algebra of multipliers (not necessarily an $L^{\infty}$ one). Vice versa, a systematic search for $\pi$-inductive algebras is one way to identify possible realizations of $\pi$.

Through Mackey's theorem and the replacement a system of imprimitivity by a strictly smaller one we get a quotient of the first realization. So it is usually enough to study maximal systems of imprimitivity (those contained in no strictly bigger one relative to the same representation). Accordingly, we restrict ourselves here to the identification of maximal inductive algebras.

This approach to the identification of realizations was proposed by Tim Steger. Although no result is known making the correspondence between realizations and inductive algebras precise, or ensuring that restriction to maximal algebras causes no loss of generality, this method precisely recovers the classical realizations in examples about matrix groups studied by Vemuri and Steger, suggesting that the failure of the approach must be connected with the existence of somewhat unnatural realizations. For other representations unexpected maximal inductive algebras might exist, associated with hitherto unknown realizations.

We review Steger and Vemuri's work more closely, highlighting the distinction between systems of imprimitivity and inductive algebras found in those cases. In [13], Vemuri dealt with the standard representation of the Heisenberg group, where he found that all maximal inductive algebras are self-adjoint, hence imprimitivity systems. For the three-dimensional Heisenberg group he confirms that no realization exists besides the standard one on $L^{2}\left(\mathbf{R}^{2}\right)$, those obtained from it through Fourier transform and (more generally) through the metaplectic group, and finally those on $L^{2}\left((\mathbf{R} / \mathbf{Z})^{2}\right)$. In [12], Steger and Vemuri dealt with representations of $\operatorname{SL}(2, \mathbf{R})$; see [7, Chapter II, 5-6]. The principal series can be realized in two different ways on $L^{2}(\partial D)$, where $D$ is the unit disc. Correspondingly, there exist exactly two maximal inductive algebras, each identifiable in the corresponding realization with the self-adjoint multiplier space $L^{\infty}(\partial D)$.

For the discrete series the natural realization is an $L^{2}$-space of holomorphic functions on $D$ and the multiplier algebra is $H^{\infty}(D)$, which is the only maximal inductive algebra. For the complementary series exactly two maximal inductive algebras exist, and they are adjoint to each other. They correspond to the two known realizations: a Sobolev space on the circle $\partial D$ and its dual. 
In this paper we take as $G$ the full automorphism group of a homogeneous tree and classify maximal inductive algebras for its spherical principal series representations, which are realized on the boundary of the tree. There is a close analogy here (see [1], [2]) with the principal series of $\operatorname{SL}(2, \mathbf{R})$, the role of $L^{2}(\partial D)$ being played now by $L^{2}(\Omega)$ (where $\Omega$ is the boundary of the tree) on which $L^{\infty}(\Omega)$ acts by multiplication.

As in Steger and Vemuri's work our results show, by ruling out the existence of unexpected maximal inductive algebras, that the list of realizations seems to be complete. A forthcoming paper will deal with further results about the principal series representation. For example, there exists exactly one more maximal inductive algebra for the midpoint representation. The new algebra is still self-adjoint but, unlike the previous examples, it is not maximal abelian. For general representations it will be shown in the same paper that restricting to the subgroup of even automorphisms does not increase the number of maximal inductive algebras.

A natural extension of this work would deal with complementary series representations $([\mathbf{1 0}])$ of the automorphism group, which are obtained by analytic continuation of the principal series. Since these representations are realized on Sobolev-like completions of the space of locally constant functions on the boundary it is easy to forecast, as in the case of $\operatorname{SL}(2, \mathbf{R})$, that non-self-adjoint maximal inductive algebras (corresponding to spaces of Sobolev space multipliers) do exist.

For cuspidal series representations $([\mathbf{1 0}])$, instead, one knows of realizations on discrete spaces. Indeed such a representation is constructed by inducing from a compact open subgroup $H$ of $G$. It remains to discover how many inductive algebras exist for a given cuspidal series representation; also whether inductive algebras other than $\ell^{\infty}(G / H)$ exist, and what sorts of realizations are associated to them.

Finally, we remark that these results ought to be extended to the principal series of $\operatorname{PGL}(2, \mathbf{F})$ (for $\mathbf{F}$ a nonarchimedean local field such as the $p$-adics), regarded as a group of automorphisms of its tree $([\mathbf{1}],[\mathbf{2}])$.

\section{Homogeneous trees and their automorphisms}

The results quoted in this section are taken from $[\mathbf{3}$, Chapter I], where detailed proofs can be found.

2.1. Chains and geodesics on trees. A tree is a connected graph with no circuits. This means that, given two vertices $y, z$, there exists exactly one finite sequence $\left\{x_{0}=y, \ldots, x_{n}=z\right\}(n \geq 1)$ such that $\left\{x_{j}, x_{j+1}\right\}$ is an edge for $j<n$ and $x_{j} \neq x_{j+2}$ for $j<n-1$. Such a sequence is said to be a chain of length $n$ and is denoted by $\left(x_{0}, x_{1}, \ldots, x_{n}\right)$ or $[y, z]$. Setting $d(y, z)=n$ if the chain connecting $y$ with $z$ has length $n$ one defines an integer-valued distance on the tree. The set $\{x \in \mathcal{X}: d(x, y) \leq k\}$ (the 
sphere of radius $n$ centered in $y)$ will be denoted by $B(y, k)$. If a reference vertex $O$ is fixed we will put $|x|:=d(x, O)$.

We shall suppose that, for some positive integer $q$, each vertex of the tree has exactly $q+1$ neighbours. Such a tree is said to be homogeneous and locally finite with degree of homogeneity $q+1$. Both the tree and its set of vertices will be denoted by $\mathcal{X}$; note that $\mathcal{X}$ is infinite if $q \geq 1$. We shall always assume that $q \geq 2$.

The concept of a chain can be generalized in a natural way by letting the index $j$ range over all of $\mathbf{N}$ or $\mathbf{Z}$. One obtains, respectively, infinite and doubly infinite chains. A doubly infinite chain is also called a geodesic.

Two infinite chains $\left(x_{i}\right)_{i=0}^{\infty}$ and $\left(y_{i}\right)_{i=0}^{\infty}$ are declared to be equivalent when, for some $n$, we have $x_{i}=y_{n+i}$ infinitely often (or, which is the same, from some $i$ on). This is indeed an equivalence relation on the set of infinite chains; the quotient space $\Omega$ is called the boundary of the tree. Given a vertex $z$, every class $\omega \in \Omega$ contains exactly one infinite chain through $z$; one can think of $\omega$ as its limit point.

Given two vertices $x, y$, we denote by $\mathcal{X}(x, y)$ the subset of $\mathcal{X}$ that can be reached by chains starting in $x$ and having $[x, y]$ as a subchain. Call $\Omega(x, y)$ the set of limit points of infinite chains contained in $\mathcal{X}(x, y)$. Varying $x, y$, and considering intersections with $\Omega$, one obtains a basis for a compact topology on $\Omega$. The family of all sets $\mathcal{X}(x, y) \cup \Omega(x, y)(x, y \in \mathcal{X})$, together with the singletons of $\mathcal{X}$, is a basis for a compact topology on $\mathcal{X} \cup \Omega$. With respect to this topology $\Omega$ is closed. Functions defined on $\Omega$ will be represented as in Figure 1. That particular example shows the function equal to $\eta$ on $\Omega(O, x)$, to $\theta$ on $\Omega(O, y)$ and to $\varepsilon$ on $\Omega(x, O) \sim \Omega(O, y)$. When no value is specified for some subset of $\Omega$, it will be understood that the function is zero there.

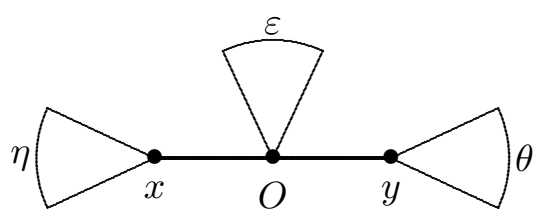

Figure 1.

2.2. The automorphism group $G$. A distance-preserving bijection of the set of vertices of $\mathcal{X}$ is called an automorphism of $\mathcal{X}$. The group of such maps will be denoted by $G$. Notice that $G$ also acts naturally on $\Omega$.

Now, given $O \in \mathcal{X}$, let $O^{\prime}$ be a neighbour of $O$. For $x \in \mathcal{X}$, let $d\left(x,\left\{O, O^{\prime}\right\}\right)$ $=\min \left(d(x, O), d\left(x, O^{\prime}\right)\right)$ and

$$
\begin{cases}\mu(\Omega(O, x))=\frac{1}{2} q^{-d\left(x,\left\{O, O^{\prime}\right\}\right)} & \text { if } x \notin\left\{O, O^{\prime}\right\} \\ \mu\left(\Omega\left(O, O^{\prime}\right)\right)=\mu\left(\Omega\left(O^{\prime}, O\right)\right)=\frac{1}{2} . & \end{cases}
$$


It is easy to see that $\mu$ can be extended to a Borel probability measure on $\Omega$ which is invariant through the subgroup $K^{\prime}$ that stabilizes $\left\{O, O^{\prime}\right\}$. In other words, given $k \in K^{\prime}$, the measure $\mu_{k}$ such that $\mu_{k}(A)=\mu\left(k^{-1} A\right)$ for every Borel set $A$ is equal to $\mu$.

Remark 1. From now on we depart slightly from the line of [3], where a different measure $\nu$ is considered. That measure is invariant through the subgroup that fixes $O$. However $\nu \cong \mu$ and every result about $\nu$ applies, with minor modifications, to the present case.

For general $g, \mu_{g}$ need not be equal to $\mu$; the argument in [3, Section II.1] nonetheless shows that $\mu_{g} \cong \mu$, and the Radón-Nikodým derivative $d \mu_{g} / d \mu$ takes only finitely many values on $\Omega$.

2.3. Spherical representations of $\boldsymbol{G}$. Fix $s \in \mathbf{R}$; for $g \in G$ let $P(g, \cdot):=$ $\left(d \mu_{g} / d \mu\right)$ and put, for $f \in \mathcal{H}:=L^{2}(\Omega, \mu)$,

$$
\pi_{s}(g) f(\omega)=P(g, \omega)^{1 / 2+i s} f\left(g^{-1} \omega\right) \quad \text { for almost every } \omega \in \Omega .
$$

The cocycle identity $P(g h, \omega)=P(g, \omega) P\left(h, g^{-1} \omega\right)$ implies that $\pi_{s}$ is a unitary representation of $G$ on $\mathcal{H}$. The family $\left\{\pi_{s}: s \in \mathbf{R}\right\}$ is called the principal spherical series of $G$.

Proposition 2.1. Let $\pi_{s}$ be a spherical representation of the principal series and $\mathcal{M}$ the algebra of multiplication operators on $\mathcal{H}$. Then $\mathcal{M}$ is a maximal $\pi_{s}$-inductive algebra.

Proof. For $\phi \in L^{\infty}(\Omega), f \in \mathcal{H}$ let $M_{\phi} f:=\phi \cdot f$. Let $(\lambda(g) \phi)(\omega)=\phi\left(g^{-1} \omega\right)$ for every $g \in G$. A computation shows that $\pi_{s}(g) M_{\phi} \pi_{s}\left(g^{-1}\right)=M_{\lambda(g) \phi}$, so that $\mathcal{M}$ is $\pi_{s}$-inductive. It is well-known that $\mathcal{M}$ is maximal commutative (see [11, Proposition 4.7.6]), hence maximal $\pi_{s}$-inductive.

We recall now (see [3, Section II.3]) that $\pi_{s}$ is equivalent to $\pi_{-s}$ for every value of $s$. Let $I_{s}$ be a unitary intertwining operator: then $\left.\pi_{-s}\right|_{K^{\prime}}$ fixes $I_{s} \mathbf{1}$ since $\left.\pi_{s}\right|_{K^{\prime}}$ fixes $\mathbf{1}$. So $I_{s} \mathbf{1}$ is constant, and by unitarity its modulus is one. Since we are mainly interested in conjugation by $I_{s}$, we may well assume that $I_{s} \mathbf{1}=\mathbf{1}$ for every $s$. With this normalization $I_{-s}=I_{s}^{-1}$ for every $s$. Since $\pi_{s}(g) \mathbf{1}=P^{1 / 2+i s}(g, \cdot)$, the intertwining property of $I_{s}$ also gives

$$
I_{s}\left(P^{1 / 2+i s}(g, \omega)\right)=P^{1 / 2-i s}(g, \omega) \quad \text { for almost every } \omega \in \Omega .
$$

It follows from Proposition 2.1 that, for every $s$, the algebra $I_{-s} \mathcal{M} I_{s}$ is maximal $\pi_{s}$-inductive. We will see in Corollary 3.4 that for most values of $s$ the new $\pi_{s}$-inductive algebra is different from $\mathcal{M}$, though clearly isomorphic to it. 


\section{Classification of maximal inductive algebras}

3.1. Operators that transform according to a character. As will soon be clear, the classification of maximal $\pi_{s}$-inductive algebras can be accomplished by studying their elements that transform in a simple way under conjugation by $\pi_{s}$ (when the latter is restricted to certain compact subgroups of $G$ ).

Definition 1. Fix two adjacent vertices $O, O^{\prime}$; let $K:=K_{O} \cap K_{O^{\prime}}$ (the compact subgroup of automorphisms that fix both $O$ and $O^{\prime}$ ). Furthermore, fix $j \in G$ that exchanges $O$ with $O^{\prime}$, and let $K^{\prime}$ be, as in Section 2.2, the compact subgroup of automorphisms that stabilize $\left\{O, O^{\prime}\right\}$. Then $K^{\prime}=$ $K \cup j K$.

For $k \in K^{\prime}$, we put

$$
\chi(k):=\left\{\begin{aligned}
1 & \text { if } k O=O, \\
-1 & \text { if } k O=O^{\prime} .
\end{aligned}\right.
$$

Finally, we say that an operator $T \in \mathcal{B}(\mathcal{H})$ transforms according to $\chi$ when

$$
\pi_{s}(k) T \pi_{s}\left(k^{-1}\right)=\chi(k) T \quad \text { for every } k \in K^{\prime} .
$$

If $\pi_{s}(k) T \pi\left(k^{-1}\right)=T$ for every $k \in K^{\prime}$, we simply call $T$ a $K^{\prime}$-invariant operator. Denote by $\mathcal{F}$ the subalgebra of $K^{\prime}$-invariant operators and by $\mathcal{E}$ the subspace of operators that transform according to $\chi$.

It is easy to see that

$$
\begin{aligned}
\mathcal{M} \cap \mathcal{F} & =\mathbf{C} M_{1}=\mathbf{C} I, \\
\mathcal{M} \cap \mathcal{E} & =\mathbf{C} M_{W},
\end{aligned}
$$

where $W:=\mathbf{1}_{\Omega\left(O, O^{\prime}\right)}-\mathbf{1}_{\Omega\left(O^{\prime}, O\right)}$. Indeed, for $\phi \in L^{\infty}$, the condition $M_{\phi} \in \mathcal{F}$ means that, for any $k \in K^{\prime}, \phi(k \omega)=\phi(\omega)$ almost everywhere. Let $d k$ be the normalized Haar measure on $K^{\prime}$. Then, by Fubini's Theorem,

$$
\begin{aligned}
0=\int_{K^{\prime}} \int_{\Omega}|\phi(k \omega)-\phi(\omega)| d \mu d k & =\int_{\Omega} \int_{K^{\prime}}|\phi(k \omega)-\phi(\omega)| d k d \mu \\
& \geq \int_{\Omega}\left|\phi(\omega)-\int_{K^{\prime}} \phi(k \omega) d k\right| d \mu .
\end{aligned}
$$

The last integral over $K^{\prime}$ is independent of $\omega$ by transitivity, so $\phi$ must be constant. The second statement is proved by applying the first one to the function $W \cdot \phi$.

Lemma 3.1. Let $\mathcal{A}$ be a maximal $\pi_{s}$-inductive algebra. If $M_{W} \in \mathcal{A}$, then $\mathcal{A}=\mathcal{M}$.

Proof. Let $T \in \mathcal{A}$ and $g \in G$; since $\mathbf{1}_{\Omega\left(O^{\prime}, O\right)}=\frac{1}{2}(W+I)$ we have

$$
\left[M_{\lambda(g) \mathbf{1}_{\Omega\left(O^{\prime}, O\right)}}, T\right]=\left[M_{\mathbf{1}_{\Omega\left(O^{\prime}, O\right)}}, \pi\left(g^{-1}\right) T \pi(g)\right]=0 .
$$


Translates of $\mathbf{1}_{\Omega\left(O^{\prime}, O\right)}$ span a dense subspace of $C(\Omega)$, so $T$ commutes with the subalgebra

$$
\mathcal{N}=\left\{M_{\phi}: \phi \in C(\Omega)\right\}
$$

of $\mathcal{M}$. But $\mathcal{N}^{\prime}=\mathcal{M}$ (see again $[\mathbf{1 1}$, Section 4.7]), hence $\mathcal{A}=\mathcal{M}$ by maximality.

The next result describes, for any maximal inductive algebra $\mathcal{A}$, the subspaces $\mathcal{A} \cap \mathcal{E}$ and $\mathcal{A} \cap \mathcal{F}$. This is the crucial step towards the classification of maximal inductive algebras, which will be summarized in Section 3.3.

Theorem 3.2. Let $\pi_{s}$ be a representation of the principal spherical series, with $\operatorname{Re} q^{i s} \neq 0$, and let $\mathcal{A}$ be a maximal $\pi_{s}$-inductive algebra. Then:

(i) $\mathcal{A} \cap \mathcal{F}=\mathbf{C} I$.

(ii) Either $\mathcal{A} \cap \mathcal{E} \subseteq \mathbf{C} M_{W}$ or $\mathcal{A} \cap \mathcal{E} \subseteq \mathbf{C}\left(I_{s} M_{W} I_{-s}\right)$.

Remark 2. One could be tempted to prove part (ii) only and then deduce part (i) on the following grounds: if $T_{1} \in \mathcal{E}$ and $T_{2} \in \mathcal{F}$, then $T_{1} T_{2} \in \mathcal{E}$. Unfortunately $T_{2}$ and $T_{1}$ need not belong to the same maximal inductive algebra, so in general part (ii) does not apply to $T_{1} T_{2}$. The reasoning can be justified because any maximal inductive algebra containing $T_{2}$ must also contain some nontrivial element of $\mathcal{E}$, but our proof of this fact (Theorem 4.1) relies on Theorem 3.2.

Remark 3. It will be clear that part (i) holds regardless of the value of $\operatorname{Re} q^{i s}$.

In the proof of Theorem 3.2 we will use the following orthogonal decomposition of the Hilbert space $\mathcal{H}$, which provides an easy description of the relevant operators as infinite matrices:

Definition 2. For $n \geq 1$, let $\mathcal{M}_{n}$ be the subspace of $\mathcal{H}$ generated by the set

$$
\left\{\mathbf{1}_{\Omega(O, x)}: d\left(x,\left\{O, O^{\prime}\right\}\right)=n\right\} .
$$

In other words, $\mathcal{M}_{n}$ is the subspace of locally constant functions on $\Omega$ that depend only of the first $n$ steps from $\left\{O, O^{\prime}\right\}$.

Moreover, let

$$
\begin{aligned}
\mathcal{H}_{n+1} & =\mathcal{M}_{n+1} \ominus \mathcal{M}_{n} \quad(n \geq 1), \\
\mathcal{H}_{0} & =\mathbf{C} 1 \\
\mathcal{K} & =\mathbf{C} W:=\mathbf{C}\left(\mathbf{1}_{\Omega\left(O, O^{\prime}\right)}-\mathbf{1}_{\Omega\left(O^{\prime}, O\right)}\right), \\
\mathcal{M}_{0} & =\mathcal{H}_{0} \oplus \mathcal{K}, \\
\mathcal{H}_{1} & =\mathcal{M}_{1} \ominus\left(\mathcal{H}_{0} \oplus \mathcal{K}\right) .
\end{aligned}
$$

See Figure 2 for examples. Notice that the mean of elements of $\mathcal{H}_{n}(n \geq 1)$ on each half of the boundary is zero while the function $W$, which generates $\mathcal{K}$, is orthogonal to $\mathbf{1}$ but not to $\mathbf{1}_{\Omega\left(O, O^{\prime}\right)}$ and $\mathbf{1}_{\Omega\left(O^{\prime}, O\right)}$. 


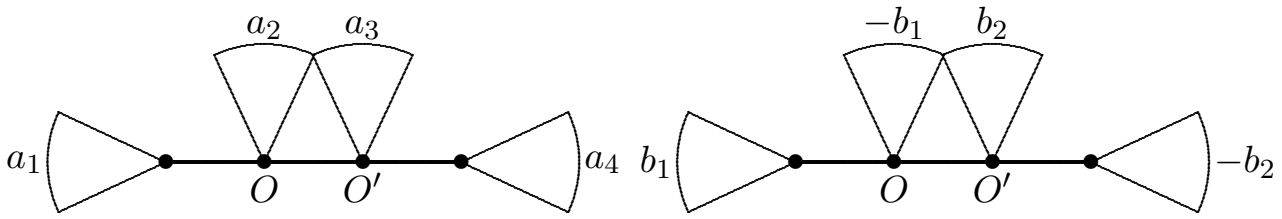

Figure 2. General elements of $\mathcal{M}_{1}$ and of $\mathcal{H}_{1}(q=2)$.

We let $\mathcal{H}_{n}^{+}$be the subspace of functions of $\mathcal{H}_{n}$ whose support lies in $\Omega\left(O^{\prime}, O\right)$. Now $\operatorname{dim} \mathcal{H}_{0}=\operatorname{dim} \mathcal{K}=1$, while $\operatorname{dim} \mathcal{M}_{n}=2 q^{n}$ and $\operatorname{dim} \mathcal{H}_{n}=$ $2(q-1) q^{n-1}(n \geq 1)$. It follows that, for $n \geq 1, \mathcal{M}_{n}=\mathcal{H}_{0} \oplus \mathcal{K} \oplus \bigoplus_{j=1}^{n} \mathcal{H}_{j}$. Since locally constant functions are dense in $\mathcal{H}$ we conclude that

$$
\mathcal{H}=\mathcal{H}_{0} \oplus \mathcal{K} \oplus \bigoplus_{j=1}^{\infty} \mathcal{H}_{j} .
$$

We compute, for later use, the action of the intertwining operator on $\mathcal{K}$ :

Lemma 3.3. Let $W$ be as in (3.4) and let $I_{s}$ be, as in Section 2.3, the unitary intertwiner of $\pi_{s}$ and $\pi_{-s}$ such that $I_{s} \mathbf{1}=\mathbf{1}$. Let

$$
\psi(s):=\frac{q-1+2 \operatorname{Im} q^{1 / 2+i s}}{q-1-2 \operatorname{Im} q^{1 / 2+i s}} .
$$

Then $I_{s} W=\psi(s) W$.

Proof. We have

$$
I_{s}\left(\frac{d \mu_{g}}{d \mu}\right)^{1 / 2+i s}=I_{s}\left(\pi_{s}(g) \mathbf{1}\right)=\pi_{-s}(g) I_{s}^{-1} \mathbf{1}=\pi_{-s}(g) \mathbf{1}=\left(\frac{d \nu_{g}}{d \nu}\right)^{1 / 2-i s} .
$$

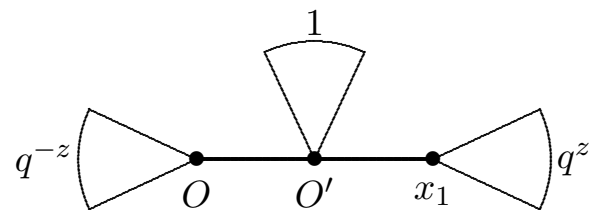

Figure 3. $d \mu_{g} / d \mu$.

Take a geodesic $\left(x_{i}\right)_{i \in \mathbf{Z}}$ containing $O$ and $O^{\prime}$, and suppose that $g x_{i}=x_{i+1}$ for all $i$. In this case $g$ is called a one step translation along $\left[O, O^{\prime}\right]$. The Radón-Nikodým derivative $d \mu_{g} / d \mu$ can be evaluated as in [3, Chapter II]; see Figure 3 for an example with $g O^{\prime}=O$. The sum of all the functions $\left(d \mu_{g} / d \mu\right)^{1 / 2+i s}$, with $g$ ranging over the set of translations just described, equals $\left(q-1-2 \operatorname{Im} q^{1 / 2+i s}\right) W$, and the thesis follows from the two previous equalities.

Corollary 3.4. With the same notation as in Lemma $3.3, I_{-s} \mathcal{M} I_{s} \neq \mathcal{M}$ if and only if $q^{2 i s} \neq 1$. 
Proof. Let $T=I_{-s} M_{W} I_{s}$, and suppose that $T=M_{f}$. Since $I_{s} \mathbf{1}=\mathbf{1}$ by definition, Lemma 3.3 gives $T \mathbf{1}=\psi(-s) W$, hence $f=\psi(-s) W$. On the other hand $T W=I_{-s} M_{W} \psi(s) W=\psi(s) I_{-s} \mathbf{1}=\psi(s) \mathbf{1}$, because $W^{2}=\mathbf{1}$. So we also get $f=\psi(s) W$. But $\psi(s) \neq \psi(-s)$ when $q^{2 i s} \neq 1$. This contradiction shows that $I_{s} M_{W} I_{-s}$ is not a multiplication operator.

Conversely, if $q^{2 i s}=1$, the same reasoning shows that $I_{s} M_{W} I_{-s} \in \mathcal{M}$. Subtracting $I$ we get $I_{s} M_{1_{\Omega\left(O^{\prime}, O\right)}} I_{-s} \in \mathcal{M}$. Conjugating by $\pi_{s}$ we find that $I_{s} M_{\lambda(g) \mathbf{1}_{\Omega\left(O^{\prime}, O\right)}} I_{-s} \in \mathcal{M}$ for every $g$. We conclude that $I_{s} \mathcal{M} I_{-s}=\mathcal{M}$ as in the proof of Lemma 3.1.

Definition 3. For two representations $\pi_{1}, \pi_{2}$ of a group $H$, let $c_{H}\left(\pi_{1}, \pi_{2}\right)$ denote the dimension of the space of unitary intertwining operators.

Let

$$
\pi_{\mathcal{K}}:=\left.\operatorname{Res}\left(\pi_{s}, K^{\prime}\right)\right|_{\mathcal{K}} \quad \text { and } \quad \pi_{n}:=\left.\operatorname{Res}\left(\pi_{s}, K^{\prime}\right)\right|_{\mathcal{M}_{n}} \quad(n \geq 0) .
$$

Lemma 3.5. The representations $\pi_{\mathcal{K}}, \pi_{n}(n \geq 0)$ defined as in (3.8) are irreducible and inequivalent.

Proof. Let $\tau$ be a finite chain of length $n$, such that $\tau \cap\left\{O, O^{\prime}\right\}=\left\{O^{\prime}\right\}$, and let $K_{\tau}$ be the subgroup of $K^{\prime}$ which fixes every vertex of $\tau$. Then $\pi_{n}$ is isomorphic to the representation $\operatorname{Ind}\left(K_{\tau}, K^{\prime}, \mathbf{1}\right)$ induced on $K^{\prime}$ from the trivial representation 1 of $K_{\tau}$. By Frobenius' reciprocity theorem ([6, VI.11, Theorem 7]),

$$
c_{K^{\prime}}\left(\operatorname{Ind}\left(K_{\tau}, K^{\prime}, \mathbf{1}\right), \operatorname{Ind}\left(K_{\tau}, K^{\prime}, \mathbf{1}\right)\right)=c_{K_{\tau}}\left(\mathbf{1}, \pi_{n}\right)
$$

and the last number is the dimension of the subspace of $\left.\pi_{n}\right|_{K_{\tau}}$-invariant elements of $\mathcal{M}_{n}$. This subspace is easily seen to be $n+2$-dimensional. So we have proved that $n+2=c\left(\pi_{n}, \pi_{n}\right)$. Since $\pi_{n}=\left.\left.\pi\right|_{\mathcal{K}} \oplus \bigoplus_{j=0}^{n} \pi\right|_{\mathcal{H}_{j}}$, it follows that the summands are irreducible and inequivalent.

Definition 4. For $n \in \mathbf{N}$ define $\iota_{n}$ to be the projection on $\mathcal{H}_{n}$, and $\iota_{\mathcal{K}}$ to be the projection on $\mathcal{K}$. Given an operator $T \in \mathcal{B}(\mathcal{H})$, let $T_{m, n}:=\iota_{m} T \iota_{n}^{*}$. If one thinks of $T$ as an infinite matrix $A$, then $T_{n, n}$ corresponds to a block $A_{m, n}$. Define similarly, for every $n$, the operators $T_{\mathcal{K}, n}, T_{n, \mathcal{K}}$ and the blocks $A_{\mathcal{K}, n}, A_{\mathcal{K}, n}$ of $A$.

When $T$ belongs to an inductive algebra, some information about its matrix $A$ can be obtained from Lemma 3.5.

Proposition 3.6. Let $\mathcal{A} \subset \mathcal{B}(\mathcal{H})$ be a maximal inductive algebra and $T \in$ $\mathcal{A} \cap \mathcal{E}$. Consider the matrix $A$ of $T$ and its finite blocks $A_{i, j}$ as in Definition 4. Then:

(i) For $i, j \geq 1, A_{i, j}$ is a multiple of $\left.M_{W}\right|_{\mathcal{H}_{i}}$ when $i=j$, and zero otherwise.

(ii) $A_{i, \mathcal{K}}, A_{\mathcal{K}, i}$ are zero unless $i=0$. 
Proof. Since $T$ transforms according to $\chi$, for every $n, m \geq 1 \iota_{n} T \iota_{m}^{*}$ intertwines $\left.\operatorname{Res}\left(\pi_{s}, K^{\prime}\right)\right|_{\mathcal{H}_{n}}$ with $\left.\chi \otimes \operatorname{Res}\left(\pi_{s}, K^{\prime}\right)\right|_{\mathcal{H}_{m}}$, while $\iota_{j} T \iota_{\mathcal{K}}^{*}$ intertwines $\left.\operatorname{Res}\left(\pi_{s}, K^{\prime}\right)\right|_{\mathcal{H}_{j}}$ with $\left.\chi \otimes \operatorname{Res}\left(\pi_{s}, K^{\prime}\right)\right|_{\mathcal{K}}$. A similar result holds for $\iota_{\mathcal{K}} T \iota_{j}^{*}$, and the thesis follows from Lemma 3.5 and Schur's Lemma.

We are now ready to classify the operators of $\mathcal{A}$ that are $K$-invariant or transform according to the character $\chi$.

\subsection{Proof of Theorem 3.2.}

Part (i). Let $T \in \mathcal{A} \cap \mathcal{F}$. By Proposition 3.6 there exists a sequence $\left\{\lambda_{0}, \lambda_{\mathcal{K}}, \lambda_{1}, \lambda_{2}, \ldots\right\}$ such that

$$
T=\lambda_{0} I_{\mathcal{H}_{0}} \oplus \lambda_{\mathcal{K}} I_{\mathcal{K}} \oplus \bigoplus_{j \geq 1} \lambda_{j} I_{\mathcal{H}_{j}}
$$

Refer to Definition 2 for details about the subspaces of $\mathcal{H}$ involved. To prove that the relevant sequence is constant, we remark first that $\left[T, T^{*}\right]=0$, and so eigenspaces of different eigenvalues of $T$ are orthogonal. Put

$$
T^{g}:=\pi\left(g^{-1}\right) T \pi(g)
$$

and suppose that there exist $v_{l} \in \mathcal{H}_{l}, v_{m} \in \mathcal{H}_{m}$ and $g \in G$ such that

$$
\left\langle T \pi(g) v_{l}, \pi(g) v_{m}\right\rangle=\left\langle T^{g} v_{l}, v_{m}\right\rangle \neq 0 .
$$

Then $v_{m}$ is an eigenvector of $\lambda_{m}$; since $\left[T, T^{g}\right]=0, T^{g} v_{l}$ is an eigenvector of $\lambda_{l}$. Hence $\lambda_{l}=\lambda_{m}$.

Keeping this in mind, we prove that $\lambda_{0}=\lambda_{\mathcal{K}}$. By maximality $T-\lambda_{0} I \in \mathcal{A}$, so we may assume that $\lambda_{0}=0$. As in the proof of Lemma 3.3, let $g$ be a translation by $-l$ along a geodesic $\left(x_{j}\right)_{j=-\infty}^{\infty}$, with $x_{-1}=O$ and $x_{0}=O^{\prime}$, and choose $v_{l}$ as in Figure 4. Using $v_{1} \in \mathcal{H}_{1}$ as in Figure 5, write

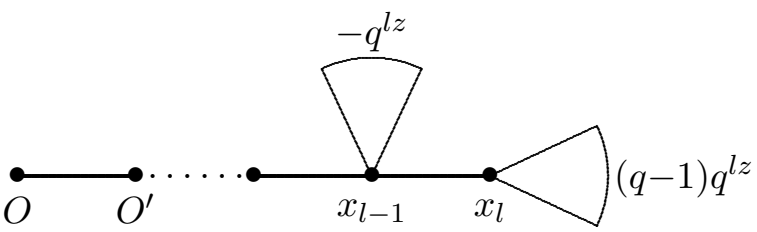

Figure 4. $v_{l} \in \mathcal{H}_{l}$.

$$
\pi(g) v_{l}=\frac{(q-1)\left(q-q^{z}\right)}{2 q} \mathbf{1}+\frac{(q-1)\left(q+q^{z}\right)}{2 q} W+\frac{q^{z}}{q} v_{1} .
$$

Since $\lambda_{0}=0$, we have

$$
T \pi(g) v_{l}=\lambda_{\mathcal{K}} \frac{(q-1)\left(q+q^{z}\right)}{2 q} W+\lambda_{1} \frac{q^{z}}{q} v_{1} .
$$




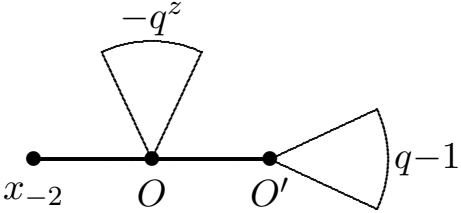

$\pi(g) v_{l}$

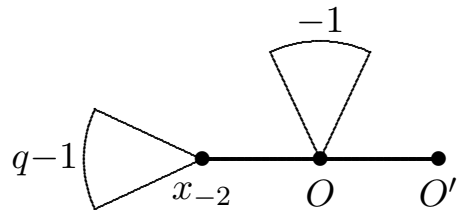

$v_{1} \in \mathcal{H}_{1}$

Figure 5 .

Then

$$
\begin{aligned}
\zeta:=\left.\left(T \pi(g) v_{l}\right)\right|_{\Omega\left(O, x_{-2}\right)} & =-\lambda_{\mathcal{K}} \frac{(q-1)\left(q+q^{z}\right)}{2 q}+\lambda_{1} \frac{(q-1) q^{z}}{q} \\
& =\frac{q-1}{2 q}\left(-\lambda_{\mathcal{K}}\left(q+q^{z}\right)+2 \lambda_{1} q^{z}\right) .
\end{aligned}
$$

Refer to Figure 6 for pictures of $\pi(g) \mathbf{1}$ and $\pi(g) W$.

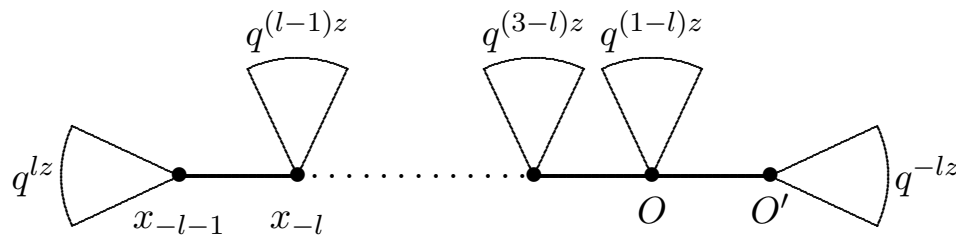

$\pi(g) \mathbf{1}$

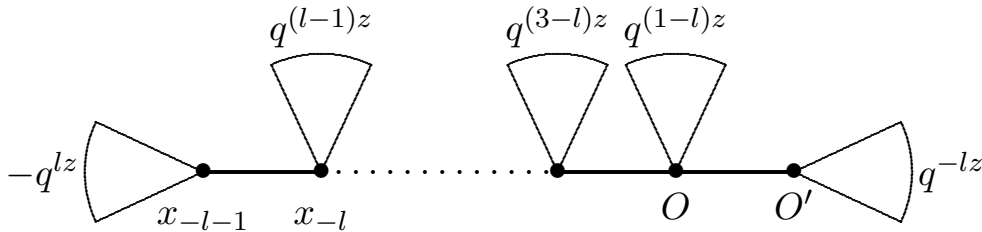

$\pi(g) W$

Figure 6.

If $\zeta \neq 0$ we can choose $v_{l}$ in such a way that $\left\langle T \pi(g) v_{l}, \pi(g) \mathbf{1}\right\rangle \neq 0$ and $\left\langle T \pi(g) v_{l}, \pi(g) W\right\rangle \neq 0$ and then conclude that $\lambda_{\mathcal{K}}=\lambda_{l}=\lambda_{0}=0$ using the reasoning explained above.

Now suppose that $\zeta=0$. Then, unless $\lambda_{\mathcal{K}}=0$, we will have

$$
\frac{\lambda_{1}}{\lambda_{\mathcal{K}}}=\frac{q+q^{z}}{2 q^{z}}=\frac{q^{1-z}+1}{2} .
$$

If this is the case then $\left(\lambda_{1} / \lambda_{\mathcal{K}}\right)^{2} \neq \lambda_{1} / \lambda_{\mathcal{K}}$, since $q>1$, so applying these same arguments to $T^{2}$ instead of $T$ we find $\lambda_{\mathcal{K}}^{2}=0$, hence $\lambda_{\mathcal{K}}=0$. 
Now we prove that $\lambda_{j}=\lambda_{0}$ for $j \geq 1$, given that $\lambda_{0}=\lambda_{\mathcal{K}}$. By maximality, again, we can subtract $\lambda_{0} I$. So we reduce to prove that $\lambda_{1}=\lambda_{2}=\cdots=0$ when $\lambda_{0}=\lambda_{\mathcal{K}}=0$. Suppose, by induction, that $\lambda_{0}=\lambda_{\mathcal{K}}=\lambda_{1}=\cdots=$ $\lambda_{l-1}=0$. We wish to show that $\lambda_{l}=0$. If we can find $g \in G, v \in \mathcal{M}_{l-1}$, and $v_{l} \in \mathcal{H}_{l}$ so that

$$
\left\langle T^{g} v, v_{l}\right\rangle=\left\langle\pi\left(g^{-1}\right) T \pi(g) v, v_{l}\right\rangle=\left\langle T \pi(g) v, \pi(g) v_{l}\right\rangle \neq 0
$$

then $T^{g} v$, like $v$, is in the 0 -eigenspace of $T$, while $v_{l}$ is in the $\lambda_{l}$-eigenspace, so $\lambda_{l}=0$.

Consider first the case $l=1$. Let $g$ be a translation one step to the left along the usual geodesic, $\left(x_{j}\right)_{j=-\infty}^{\infty}$, and choose $v$ and $v_{1}$ as in Figure 7 .

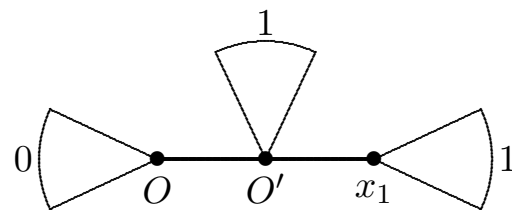

$v \in \mathcal{M}_{0}$

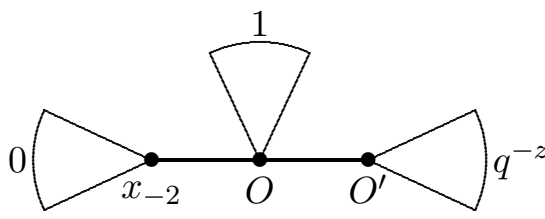

$\pi(g) v$

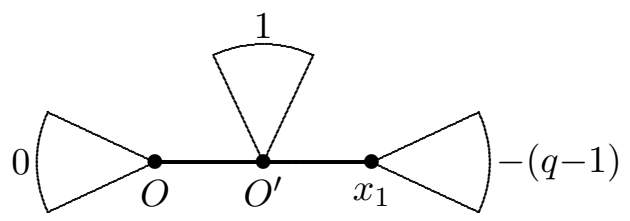

$v_{1} \in \mathcal{H}_{1}$

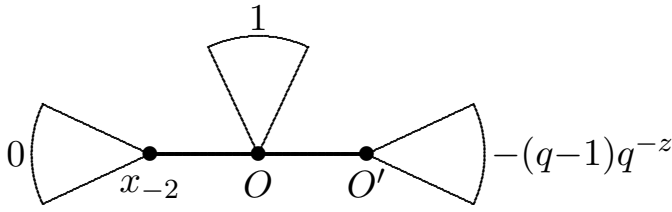

$\pi(g) v_{1}$

Figure 7. The case $l=1$.

Under the hypothesis $\lambda_{0}=\lambda_{\mathcal{K}}=0$, one easily calculates $\left\langle T \pi(g) v, \pi(g) v_{1}\right\rangle=$ $\lambda_{1}(q-1) / 2 q^{2}$. Therefore, if $\lambda_{1}$ were nonzero, the reasoning following (3.15) would show that it was zero after all.

Now consider the case of general $l$. Let $g$ be a translation by $2 l-1$ steps to the left along the usual geodesic, and choose $v$ and $v_{l}$ as in Figure 8. Figure 8 also shows the exact form of $\pi(g) v$. The form of $\pi(g) v_{l}$ is similar and all one needs to know of it is that $\left.\pi(g) v_{l}\right|_{\Omega\left(O, x_{-l-1}\right)}=0$ and $\left.\pi(g) v_{l}\right|_{\Omega\left(O, x_{-l}\right) \sim \Omega\left(O, x_{-l-1}\right)}=1$, exactly as for $\pi(g) v$. Under the hypothesis that $\left.T\right|_{\mathcal{M}_{l-1}}=0$ one calculates that $\left\langle T \pi(g) v, \pi(g) v_{l}\right\rangle=\lambda_{l}(q-1) / 2 q^{l+1}$ and then applies the reasoning following (3.15).

Part (ii). By Proposition 3.6, again, every operator $T \in \mathcal{A} \cap \mathcal{E}$ can be decomposed as

$$
T=\left.\lambda_{\mathcal{K} 0} M_{W}\left|\mathcal{H}_{0} \oplus \lambda_{0 \mathcal{K}} M_{W}\right| \mathcal{K} \oplus \bigoplus_{j \geq 1} \lambda_{j} M_{W}\right|_{\mathcal{H}_{j}}
$$




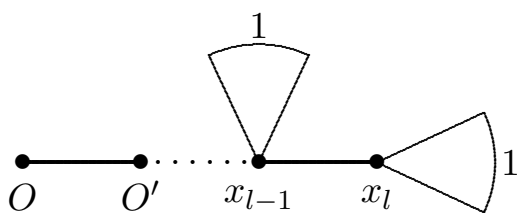

$v \in \mathcal{M}_{l-1}$

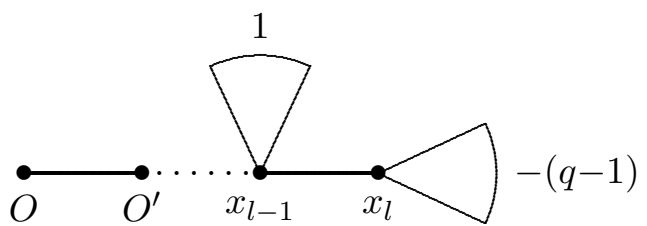

$v_{l} \in \mathcal{H}_{l}$

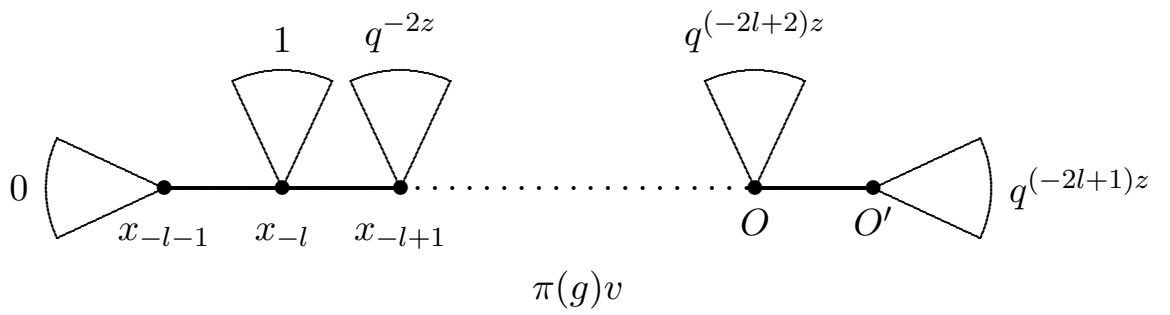

Figure 8. To prove $\lambda_{l}=0$.

for some sequence $\left\{\lambda_{\mathcal{K} 0}, \lambda_{0 \mathcal{K}}, \lambda_{1}, \lambda_{2}, \ldots\right\}$. Obviously $T \in \mathbf{C} M_{W}$ if and only if the relevant sequence is constant. We will show that this is indeed the case, up to replacing possibly $T$ with $I_{s} T I_{s}^{-1}$. By specializing Equations (1.2), (3.2) to matrix coefficients we get, for every $v \in \mathcal{H}_{1}^{+}$,

$$
\begin{aligned}
\lambda_{0 \mathcal{K}}\left\langle T^{g} \mathbf{1}, W\right\rangle & =\left\langle T^{g} T W, W\right\rangle=\left\langle T^{g} W, T^{*} W\right\rangle=\lambda_{\mathcal{K} 0}\left\langle T^{g} W, \mathbf{1}\right\rangle \\
\lambda_{0 \mathcal{K}}\left\langle T^{g} W, W\right\rangle & =\left\langle T^{g} W, T^{*} \mathbf{1}\right\rangle=\left\langle T^{g} T W, \mathbf{1}\right\rangle=\lambda_{0 \mathcal{K}}\left\langle T^{g} \mathbf{1}, \mathbf{1}\right\rangle \\
\lambda_{1}\left\langle T^{g} \mathbf{1}, v\right\rangle & =\left\langle T^{g} \mathbf{1}, T^{*} v\right\rangle=\left\langle T^{g} T \mathbf{1}, v\right\rangle=\lambda_{\mathcal{K} 0}\left\langle T^{g} W, v\right\rangle \\
\lambda_{1}\left\langle T^{g} W, v\right\rangle & =\left\langle T^{g} W, T^{*} v\right\rangle=\left\langle T^{g} T W, v\right\rangle=\lambda_{0 \mathcal{K}}\left\langle T^{g} \mathbf{1}, v\right\rangle .
\end{aligned}
$$

If

$$
\left\langle T^{g} W, v\right\rangle\left\langle T^{g} \mathbf{1}, v\right\rangle \neq 0 \quad \text { for some } g \in G, v \in \mathcal{H}_{1}^{+},
$$

we multiply the last two equations in (3.17) and get

$$
\lambda_{\mathcal{K} 0} \lambda_{0 \mathcal{K}}=\lambda_{1}^{2}
$$

Suppose now that condition (3.18) fails. Observe that, if exactly one of the factors in (3.18) (the first one, say) vanishes, Equations (3.17) give $\lambda_{1}=\lambda_{\mathcal{K} 0}=0$, so that (3.19) still holds. So we reduce to the case in which

$$
\left\langle T^{g} W, v\right\rangle=\left\langle T^{g} \mathbf{1}, v\right\rangle=0
$$

for all $v \in \mathcal{H}_{1}^{+}$and $g \in G$. In particular, let $g$ be a translation one step on the left along the usual geodesic. Take $v \in \mathcal{H}_{1}$ such that $\langle v, \pi(g) \mathbf{1}\rangle \neq 0$ and renormalize $v$ so that $\|v\|^{2}=(q-1) / 2$. Refer again to Figure 6 for a representation of $\pi(g) \mathbf{1}, \pi(g) W$. 
Put $z=1 / 2+i s$ and

$$
\alpha:=\frac{q-1}{2 q} ; \quad \beta:=\operatorname{Re} \frac{q^{z}}{q} ; \quad \gamma:=\operatorname{Im} \frac{q^{z}}{q} .
$$

Then, for a suitable $v^{\prime} \in\langle\{\mathbf{1}, W, v\}\rangle^{\perp}$,

$$
\begin{aligned}
\pi(g) \mathbf{1} & =(\alpha+\beta) \mathbf{1}+(\alpha+i \gamma) W+q^{-1}\left(q^{z}-1\right) v \\
\pi(g) W & =(\alpha-i \gamma) \mathbf{1}+(\alpha-\beta) W-q^{-1}\left(q^{z}+1\right) v \\
\pi(g) v & =(2 q)^{-1}\left\{\left(1-\overline{q^{z}}\right) \mathbf{1}+\left(1+\overline{q^{z}}\right) W-2(q-1)^{-1} v\right\}+v^{\prime} .
\end{aligned}
$$

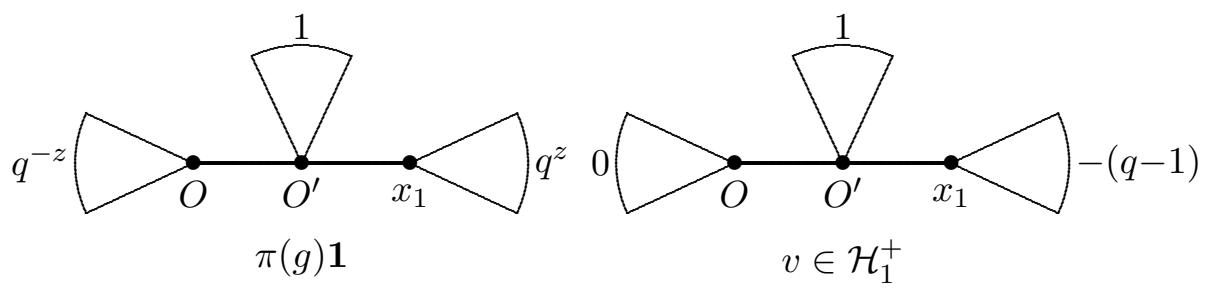

Figure 9.

Looking at the action of $T$, and assuming (3.20), we get the system

$$
\begin{aligned}
\lambda_{0 \mathcal{K}}(\alpha+i \gamma)\left(q^{z}-1\right)+\lambda_{\mathcal{K} 0}(\alpha+\beta)\left(-q^{z}-1\right)+\lambda_{1} q^{-1}\left(q^{z}-1\right) & =0 \\
\lambda_{0 \mathcal{K}}(\beta-\alpha)\left(q^{z}-1\right)+\lambda_{\mathcal{K} 0}(-\alpha+i \gamma)\left(-q^{z}-1\right)+\lambda_{1} q^{-1}\left(q^{z}+1\right) & =0 .
\end{aligned}
$$

Since $q \geq 2$, we readily obtain

$$
\begin{aligned}
& \lambda_{0 \mathcal{K}}=\lambda_{1} \frac{q^{z}\left(-2 \alpha-\overline{q^{z}} q^{-1}-q^{-1}\right)}{q^{z}-1} \\
& \lambda_{\mathcal{K} 0}=\lambda_{1} \frac{q^{z}\left(\overline{q^{z}} q^{-1}-2 \alpha-q^{-1}\right)}{q^{z}+1}
\end{aligned}
$$

(recall that $\left|q^{z}\right| \geq \sqrt{2}$ ) and finally, by a simple computation,

$$
\lambda_{0 \mathcal{K}} \lambda_{\mathcal{K} 0}=\lambda_{1}^{2} .
$$

We will now use for the first time the condition $\beta \neq 0$, that is, $\operatorname{Re} q^{1 / 2+i s} \neq$ 0 ; under this condition we will find a further, linear relation between the first coefficients of $T$. Indeed, taking $g \in G, v \in \mathcal{H}_{1}^{+}$as in (3.22), the second equation in (3.17) reads, with the same notation as in (3.21),

$$
\begin{aligned}
& \lambda_{0 \mathcal{K}}\left(\lambda_{\mathcal{K} 0}(\alpha-i \gamma)(\alpha-\beta)+\lambda_{0 \mathcal{K}}(\alpha+i \gamma)(\alpha-\beta)+\frac{\lambda_{1}}{2} q^{-2}(q-1)\left|q^{z}+1\right|^{2}\right) \\
= & \lambda_{0 \mathcal{K}}\left(\lambda_{\mathcal{K} 0}(\alpha-i \gamma)(\alpha+\beta)+\lambda_{0 \mathcal{K}}(\alpha+i \gamma)(\alpha+\beta)+\frac{\lambda_{1}}{2} q^{-2}(q-1)\left|q^{z}-1\right|^{2}\right) .
\end{aligned}
$$


If $\lambda_{0 \mathcal{K}}=0$ then $\lambda_{1}=0$ by (3.19). Moreover, by (3.22) and the first equation in (3.17),

$$
0=\lambda_{0 \mathcal{K}}\left\langle T^{g} \mathbf{1}, W\right\rangle=\lambda_{\mathcal{K} 0}\left\langle T^{g} W, \mathbf{1}\right\rangle=\lambda_{\mathcal{K} 0}^{2}|\alpha-i \gamma|^{2}
$$

that is, $\lambda_{\mathcal{K} 0}=0$, since $\gamma \in \mathbf{R}$ and $\alpha=(q-1) /(2 q)>0$ by hypothesis. Therefore, excluding the case in which $\lambda_{\mathcal{K} 0}=\lambda_{0 \mathcal{K}}=\lambda_{1}=0$ we can divide both sides in (3.26) by $\lambda_{0 \mathcal{K}}$. Since $\beta \neq 0$ (equivalently, $\left|q^{z}+1\right| \neq\left|q^{z}-1\right|$ ), the resulting relation

$$
2 \beta\left(\lambda_{\mathcal{K} 0}(\alpha-i \gamma)+\lambda_{0 \mathcal{K}}(\alpha+i \gamma)\right)=\frac{\lambda_{1}}{2} q^{-2}(q-1)\left(\left|q^{z}+1\right|^{2}-\left|q^{z}-1\right|^{2}\right)
$$

is nontrivial. Taking $\lambda_{1}$ as a parameter one sees that the solutions to the system of equations (3.28) and (3.25) are

$$
\lambda_{\mathcal{K} 0}=\lambda_{0 \mathcal{K}}=\lambda_{1}
$$

and

$$
\lambda_{\mathcal{K} 0}=\psi(s) \lambda_{1}, \quad \lambda_{0 \mathcal{K}}=(\psi(s))^{-1} \lambda_{1},
$$

with $\psi(s)$ as in (3.6). Under condition (3.29), $T=\lambda_{1} I$ on $\mathbf{C 1} \oplus \mathcal{K}$. By Lemma 3.3, $T$ satisfies (3.30) if and only if $I_{s}^{-1} T I_{s}$ satisfies (3.29). Since $\psi(s) \neq 1$ the same solution must be chosen for every $T \in \mathcal{A}$; else the product of two elements of $\mathcal{A} \cap \mathcal{E}$ would fail to be scalar, contradicting part (i).

Finally, when (3.29) holds, the same reasoning as in part (i) shows that $\lambda_{j}=\lambda_{1}$ for all $j \geq 1$. Indeed, the proof of the analogous fact for $K^{\prime}$-invariant operators only involves vectors in $\mathcal{H}_{0} \oplus \mathcal{K} \oplus \bigoplus_{j \geq 1} \mathcal{H}_{j}^{+}$. So the method also applies to operators in $\mathcal{A} \cap \mathcal{E}$, which act scalarly on any $\mathcal{H}_{j}^{+}$.

We conclude that either $T \in \mathbf{C} M_{W}$ or $I_{s} T I_{s}^{-1} \in \mathbf{C} M_{W}$.

3.3. Proof of the main classification result. Suppose that $\operatorname{Im} q^{i s} \neq 0$ and $\operatorname{Re} q^{i s} \neq 0$. Take a maximal $\pi_{s}$-inductive algebra $\mathcal{A}$ and assume, for a moment, that $\mathcal{A} \cap \mathcal{E} \neq\{0\}$. By Theorem 3.2, either $\mathcal{A}$ or $I_{-s} \mathcal{A} I_{s}$ contains a nonzero scalar multiple of $M_{W}$. Hence, by Lemma 3.1, either $\mathcal{A}=\mathcal{M}$ or $I_{-s} \mathcal{A} I_{s}=\mathcal{M}$. So, for these values of $s$, the classification of maximal inductive algebras is achieved, up to verifying that any such algebra must contain operators that transform according to $\chi$. The latter turns out to be true, but the proof - to be found in the next section - is not trivial.

\section{Nontrivial operators that transform according to $\chi$}

\subsection{Nonscalar $K$-invariant operators.}

Theorem 4.1. Let $\pi_{s}$ be a spherical representation of the principal series, with $q^{2 i s} \neq-1$, and let $\mathcal{A}$ be a maximal $\pi_{s}$-inductive algebra. Then:

(i) $\mathcal{A} \cap \mathcal{F}$ contains a nonscalar operator. 
(ii) $\mathcal{A} \cap \mathcal{E}$ contains a nonzero operator.

We prove first that (i) implies (ii). Indeed, given a nonscalar $K$-invariant element of $\mathcal{A}$, say $T$, take $j \in K^{\prime}$ such that $\chi(j)=-1$. Then, obviously:

- $T_{1}:=T+\pi(j) T \pi\left(j^{-1}\right)$ is $K^{\prime}$-invariant.

- $T_{2}:=T-\pi(j) T \pi\left(j^{-1}\right)$ transforms according to $\chi$.

Now $T_{1}, T_{2} \in \mathcal{A}$, and by Theorem $3.2, T_{1}$ must be scalar. Since $T$ is nonscalar, $T_{2}=2 T-T_{1} \neq 0$, so part (ii) of Theorem 4.1 holds.

The proof of part (i) is harder and will be accomplished in several steps. We recall that $K$ is the stabilizer of the subtree $\left\{O, O^{\prime}\right\}$, and set about proving first a weaker statement, replacing $\left\{O, O^{\prime}\right\}$ with a bigger subtree. More precisely, we fix $O \in \mathcal{X}$ and let $K_{n}(n \in \mathbf{N})$ be the compact subgroup of $G$ that stabilizes the subtree $B(O, n)=\{x \in \mathcal{X}: d(x, O) \leq n\}$ (a sphere of radius $n$ ). The normalized Haar measure on $K_{n}$ will be denoted by $d k$. Given $S \in \mathcal{A}$, we let

$$
S_{n}:=\int_{K_{n}} \pi(k) S \pi\left(k^{-1}\right) d k .
$$

Then $S_{n}$ is $K_{n}$-invariant and $S_{n} \in \overline{\mathcal{A}}^{w}=\mathcal{A}$. We know from Proposition 2.1 that $\mathbf{C} I$ is not maximal, so we can take as $S$ a nonscalar element of $\mathcal{A}$. In this case $S_{n}$ is also nonscalar for some $n$. To see this, consider two locally constant functions $\xi, \eta$ : then, for $n$ large enough, $\left.\pi\right|_{K_{n}}$ fixes both $\xi$ and $\eta$, so that $\left\langle S_{n} \xi, \eta\right\rangle=\langle S \xi, \eta\rangle$. Since locally constant functions are dense in $\mathcal{H}$ and $\left\{S_{n}\right\}$ is uniformly bounded, $S_{n}$ weakly approaches $S$ and so $S_{n}$ is nonscalar for some $n$. We conclude that part (i) of Theorem 4.1 holds when $K$ is replaced by the stabilizer of a suitable sphere.

4.2. Complete subtrees. Note that every vertex of a sphere $B$ has either one or $q+1$ neighbours in $B$. With the notation of [3, Chapter III], we can say that every vertex is either a boundary vertex or an interior vertex. This is also trivially true for the one-edge subtree. Subtrees verifying this condition are said to be complete (see Figure 10 for an example). We will now prove that part (i) of Theorem 4.1 holds for the stabilizer of a finite complete subtree $\mathcal{J}$, of more than one edge, only if it also holds for the stabilizer of some smaller complete subtree. This, by induction, will conclude the proof.

Given a complete subtree $\mathcal{J}$, we denote by $\partial \mathcal{J}$ the set of its boundary vertices. We say that a vertex $x \in \mathcal{J}$ is almost terminal if it is interior and exactly $q$ of its neighbours are boundary vertices. See again Figure 10.

Remark 4. From now on, given a terminal vertex $P$ of a complete subtree $\mathcal{J}$, we denote by $P^{\prime}$ its unique neighbour in $\mathcal{J}$. The subtree $\mathcal{J}^{\prime}$ obtained as in Figure 11 by erasing from $\mathcal{J}$ the neighbours of $P^{\prime}$ that lie in $\partial \mathcal{J}$ is also complete, and $P^{\prime} \in \partial \mathcal{J}^{\prime}$. 

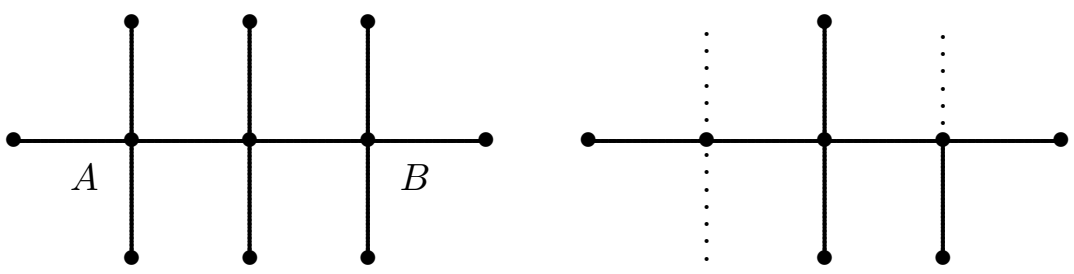

Figure 10. A complete subtree, with almost terminal vertices $A, B$, and a subtree which is not complete.

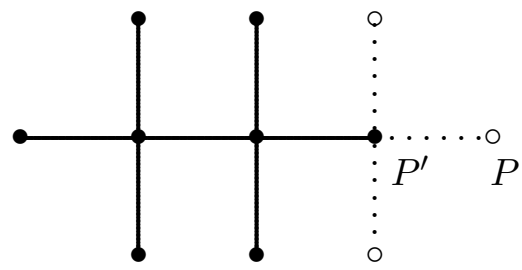

Figure 11. To obtain $\mathcal{J}^{\prime}$ from $\mathcal{J}$.

Consider the subgroup

$$
H_{P}:=\left\{g \in G: g \text { fixes } \mathcal{X}\left(P, P^{\prime}\right)\right\}
$$

of the automorphisms that only affect the vertices lying in the subtree of $\mathcal{X} \sim \mathcal{J}$ sprouting from $P$. It is shown in [3, Section III.3] that

$$
K_{\mathcal{J}}=\prod_{R \in \partial \mathcal{J}} H_{R}
$$

In particular, if $R \in \partial \mathcal{J}$ and $R \neq P$, then $H_{R}$ lies in the centralizer of $H_{P}$. When $\mathcal{J}$ is replaced by $\mathcal{J}^{\prime}$ as in Remark 4, formula (4.3) becomes

$$
K_{\mathcal{J}^{\prime}}=\left(\prod_{\substack{R \in \partial \mathcal{J} \\ d\left(R, P^{\prime}\right)>1}} H_{R}\right) \times H_{P^{\prime}}
$$

of course, all the relevant subgroups are still defined by (4.2), but with respect to the new subtree $\mathcal{J}^{\prime}$.

It follows from (4.4) that, given a $K_{\mathcal{J}}$-invariant operator $T$,

$$
\widetilde{T}:=\int_{H_{P}^{\prime}} k(T) d k,
$$

defined as in (4.1) is $K_{\mathcal{J}^{\prime}}$-invariant. Moreover, if $T$ lies in some maximal inductive algebra $\mathcal{A}$, then also $\widetilde{T} \in \mathcal{A}$. The trouble is that starting from a nonscalar operator $T$ does not guarantee that $\widetilde{T}$ will be nonscalar. We will show, however, that this is true for a suitable choice of $P$ in $\partial \mathcal{J}$; to this purpose we will describe more precisely the nonscalar $K_{\mathcal{J}}$-invariant elements of maximal inductive algebras. 
4.3. The Hilbert spaces $\mathcal{H}^{\boldsymbol{P}}$. Recall from (2.1) the definition of the subsets $\mathcal{X}\left(P_{1}, P_{2}\right) \subset \mathcal{X}$ for $P_{1}, P_{2} \in \mathcal{X}$. Consider a complete subtree $\mathcal{I}$, and associate to every $P \in \partial \mathcal{I}$ a sequence of subspaces of $\mathcal{H}$ in the following way:

$$
\begin{aligned}
\mathcal{H}^{P} & :=\left\{f \in \mathcal{H}: f=f \cdot \mathbf{1}_{\Omega\left(P^{\prime}, P\right)}\right\}, \\
\mathcal{H}_{0}^{P} & :=\mathcal{M}_{0}^{P}=\mathbf{C} \mathbf{1}_{\Omega\left(P^{\prime}, P\right)}, \\
\mathcal{M}_{n}^{P} & :=\left\langle\left\{\mathbf{1}_{\Omega\left(P^{\prime}, Q\right)}: Q \in \mathcal{X}\left(P^{\prime}, P\right) \text { and } d(Q, P)=n\right\}\right\rangle \quad(n \geq 1), \\
\mathcal{H}_{n+1}^{P} & =\mathcal{M}_{n+1}^{P} \ominus \mathcal{M}_{n}^{P} \quad(n \geq 1) .
\end{aligned}
$$

This construction should be compared with (3.5); one can say that $\mathcal{M}_{n}^{P}$ is the space of locally constant functions, with support in $\Omega\left(P^{\prime}, P\right)$, that only depend on the first $n$ steps along some finite chain in $\mathcal{X}\left(P^{\prime}, P\right)$. Note that definition (4.6) does depend on the choice of the subtree.

As in the reasoning following (3.5), we see that

$$
\mathcal{H}^{P}=\bigoplus_{j=0}^{\infty} \mathcal{H}_{j}^{P} .
$$

From this decomposition we will get more information about the action of $K_{\mathcal{I}}$-invariant operators on the subspaces $\left\{\mathcal{H}^{P}: P \in \partial \mathcal{I}\right\}$.

Lemma 4.2. Take a finite complete subtree $\mathcal{I}$ and two vertices $P, Q \in \partial \mathcal{I}$.

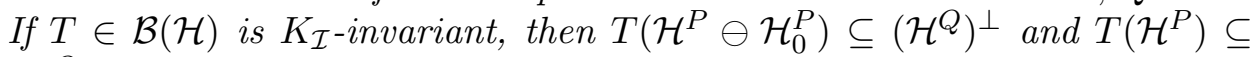
$\left(\mathcal{H}_{1}^{Q}\right)^{\perp}$.

Proof. Consider $H_{P}$ as in (4.2). Then $\mathcal{H}^{P} \ominus \mathcal{H}_{0}^{P}$ is generated by

$$
\left\{v \in \mathcal{H}^{P}: \pi(h) v=-v \text { for some } h \in H_{P}\right\} .
$$

At the same time, $\pi(h) w=w$ for all $w \in \mathcal{H}^{Q}$ and $h \in H_{P}$. Thus any $v$ as in (4.8) is orthogonal to $\mathcal{H}^{Q}$. The second inclusion follows by applying the first to $T^{*}$.

\subsection{The inductive step.}

Theorem 4.3. Let $\mathcal{A}$ be a maximal inductive algebra, and $\mathcal{I} \subset \mathcal{X}$ a finite complete subtree with at least two edges, such that $\mathcal{A}$ contains a nonscalar $K_{\mathcal{I}}$-invariant operator. Then, for some complete subtree $\mathcal{J} \subsetneq \mathcal{I}$, there exists in $\mathcal{A}$ a nonscalar $K_{\mathcal{J}}$-invariant operator. In other words, $\mathcal{I}$ is not minimal among the complete subtrees verifying Theorem 4.1.

The proof of Theorem 4.3 will take up the remainder of the paper, and will be divided into several steps. To begin with, we deduce from (4.7) and Lemma 4.2 that, for any nonscalar $K_{\mathcal{I}}$-invariant operator $T$, exactly one of the following holds:

(a) For every $P \in \partial \mathcal{J}$ there exists $\lambda_{P} \in \mathbf{C}$ such that $\left.T\right|_{\mathcal{H}^{P^{\prime}}}=\lambda_{P} I_{\mathcal{H}^{P^{\prime}}}$, but $P \mapsto \lambda_{P}$ is not constant. 
(b) Every subspace $\mathcal{H}^{P^{\prime}}$ is $T$-invariant, but the restriction of $T$ to some $\mathcal{H}^{P^{\prime}}$ is nonscalar.

(c) $T \mathcal{H}^{P^{\prime}} \not \perp \mathcal{H}^{Q^{\prime}}$ for some pair of different terminal vertices $P, Q$. In this case $\left\langle T \mathbf{1}_{\Omega\left(P^{\prime}, P\right)}, T \mathbf{1}_{\Omega\left(Q^{\prime}, Q\right)}\right\rangle \neq 0$.

Case (b) is easily dealt with. Indeed, suppose $P \in \partial \mathcal{I}$ is such that $\left.T\right|_{\mathcal{H}^{P}}$ is nonscalar. Let $H$ be the subgroup of $G$ that fixes all vertices in $\mathcal{X}\left(P^{\prime}, P\right)$. Then

$$
T^{\sharp}:=\int_{H} \pi(h) T \pi\left(h^{-1}\right) d h
$$

agrees with $T$ on $\mathcal{H}^{P}$. Consequently, $T^{\sharp}$ is nonscalar. Moreover, since $T^{\sharp}$ is fixed by $H$ and by $H_{P}$, it is fixed by $K_{\left\{P, P^{\prime}\right\}}$. So $T^{\sharp}$ and the nontrivial complete subtree $\mathcal{I} \sim B\left(P^{\prime}, 1\right) \cap \partial \mathcal{I}$ (as in Figure 11) satisfy the thesis.

For case (a), suppose first that $\mathcal{I}$ is not a sphere of radius one. Take $Q \in \partial \mathcal{I}$ and its almost terminal neighbour $Q^{\prime} ;$ put $\mathcal{J}:=\mathcal{I} \sim\left(B\left(Q^{\prime}, 1\right) \cap \partial \mathcal{I}\right)$. Then $\mathcal{J}$ is a nontrivial complete subtree. Take $H_{Q}, H_{Q^{\prime}}$ and $\widetilde{T}$ as in (4.2) and (4.5). Since $T$ acts as a scalar on each subspace $\mathcal{H}^{P}(P \in \partial \mathcal{I})$, we have $\widetilde{T} \mathcal{H}^{Q^{\prime}} \subseteq \mathcal{H}^{Q^{\prime}}$ and

$$
\left.\widetilde{T}\right|_{\mathcal{H}^{Q^{\prime}}}=\frac{1}{q}\left(\sum_{R \in \partial \mathcal{I} \cap B\left(Q^{\prime}, 1\right)} \lambda_{R}\right) I_{\mathcal{H}^{Q^{\prime}}},
$$

so that $\widetilde{T}$ acts as a scalar on $\mathcal{H}^{R}$ for every $R$ in the boundary of the smaller complete subtree $\mathcal{J}$. As remarked above, $\widetilde{T}$ may be scalar, but in that case

$$
\sum_{R \in \partial \mathcal{I} \cap B\left(Q^{\prime}, 1\right)} \lambda_{R}=q \beta \quad \text { for every } \beta \in\left\{\lambda_{P}: P \in \partial \mathcal{I}\right\} .
$$

If $\mathcal{I}$ is a sphere of radius one, with interior vertex $O$, consider $P \in \partial \mathcal{I}$ and the subgroup $K_{O, P}$ that fixes $O$ and $P$. Construct $\widetilde{T}$ as in (4.5). If $\widetilde{T}$ is scalar then $\lambda_{P}=(1 / q) \sum_{R \in \partial I ; R \neq P} \lambda_{R}$. This must fail for some $P \in \partial \mathcal{I}$, because $P \mapsto \lambda_{P}$ is not constant on $\partial \mathcal{I}$. So only case (c) remains to be considered.

4.5. Proof of Theorem 4.3 in case (c). If $\mathcal{I}$ satisfies the additional hypothesis that there exist at least three almost terminal vertices $P^{\prime}, Q^{\prime}, R^{\prime}$, the proof still runs easily. Indeed, let $P, Q, R$ be boundary neighbours of $P^{\prime}, Q^{\prime}, R^{\prime}$, respectively. Then the operator

$$
\hat{T}:=\int_{H_{R^{\prime}}} \pi(h) T \pi\left(h^{-1}\right) d h,
$$

defined as in (4.2), but relatively to the subtree $\mathcal{I} \sim\left(B\left(R^{\prime}, 1\right) \cap \partial \mathcal{I}\right)$, is invariant with respect to the stabilizer of some proper complete subtree;

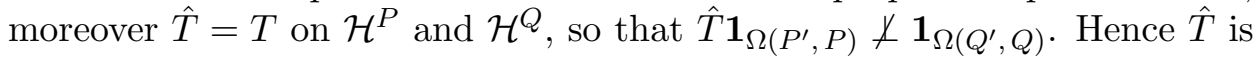
nonscalar. 
In order to achieve the proof of Theorem 4.3 we must finally consider the finite complete subtrees which fail to satisfy the additional hypothesis stated just before (4.12). It is clear that any such subtree belongs to one of the following classes:

(i) unit spheres,

(ii) unions of pairs of unit spheres, with centers at distance 1, or

(iii) unions of families of unit spheres, with centers on a given chain (other than an edge).

We first deal with case (ii). Let $O$ be an interior vertex of the subtree. Call $P_{1}, P_{s}, \ldots, P_{2 q}$ the boundary vertices of $\mathcal{I}$, with $d\left(P_{i}, O\right)>d\left(P_{i}, O^{\prime}\right)$ if and only if $i \leq q$; let $f_{i}:=\mathbf{1}_{\Omega\left(O, P_{i}\right)}$. We know from Lemma 4.2 that $T$ acts on the subspace of $\mathcal{H}$ generated by $\left\{f_{1}, f_{2}, \ldots, f_{2 q}\right\}$. With respect to this basis $T$ is represented by a nonscalar matrix

$$
A:=\left(\begin{array}{cc}
B & C \\
D & E
\end{array}\right) .
$$

If, for example, $\left\langle T f_{i}, f_{j}\right\rangle \neq 0$ for some $i, j \leq q(i \neq j)$, then, just as in the first remark in this subsection, we see that the operator

$$
\widetilde{T}:=\int_{H_{O^{\prime}}} \pi(h) T \pi\left(h^{-1}\right) d h
$$

is still nonscalar and is invariant through the smaller subgroup that fixes the sphere of radius one with center in $O^{\prime}$. The same reasoning applies if $T f_{h} \not \perp f_{k}$ with $h, k \geq q+1(h \neq k)$. So we restrict to the case in which the blocks $B$ and $D$ are both diagonal. We consider $\widetilde{T}$ as in (4.14) and compute its matrix $\widetilde{A}$ with respect to $\left\{f_{1}, f_{2}, \ldots, f_{2 q}\right\}$. Since $H_{O^{\prime}}$ acts on $\left\{f_{1}, f_{2}, \ldots, f_{2 q}\right\}$ as a permutation group, it turns out, with the notation of (4.13), that

$$
\widetilde{A}=\left(\begin{array}{cc}
\widetilde{B} & \widetilde{C} \\
\widetilde{D} & \widetilde{E}
\end{array}\right)
$$

with

$$
\begin{aligned}
\widetilde{B} & =q^{-1}\left(B_{11}+\cdots+B_{q q}\right) I_{q}, \\
\widetilde{E} & =E, \\
\widetilde{C}_{i j} & =q^{-1} \sum_{h} C_{h j} \quad \text { for every } i, j, \\
\widetilde{D}_{i j} & =q^{-1} \sum_{h} D_{i h} \quad \text { for every } i, j .
\end{aligned}
$$

By replacing $H_{O^{\prime}}$ with $H_{O}$ in (4.14) we obtain yet another element $\widetilde{T}^{\prime}$ of $\mathcal{A}$. It is easy to see that its matrix $\widetilde{A}^{\prime}$ satisfies an analogue of (4.16) (where $B$ 
and $C$ interchange their roles, respectively, with $E$ and $D$ ). If $\widetilde{T}$ and $\widetilde{T}^{\prime}$ are both scalar, we obtain from (4.16) the following conditions about $A$ :

(i) $B=E=\lambda I_{q}$ for some $\lambda \in \mathbf{C}$.

(ii) Each row and column in $C$ and $D$ adds up to zero (hence $\operatorname{det} C=$ $\operatorname{det} D=0)$.

We digress for a moment to prove the following simple result:

Lemma 4.4. Let $C$ be $a q \times q$ complex matrix such that every row and every column adds up to zero. For $\sigma, \tau \in S_{q}$, the group of permutations on $q$ objects, let $C^{\sigma, \tau}$ be the matrix such that $\left(C^{\sigma, \tau}\right)_{i j}=C_{\sigma(i) \tau(j)}$. Suppose, finally, that $C C^{\sigma, \tau}=0$ for every $\sigma, \tau$. Then $C=0$.

Proof. By hypothesis

$$
\sum_{i=1}^{q} C_{h i} C_{\sigma(i) \tau(j)}=0 \quad \text { for every } h, j \in\{1, \ldots, q\}, \sigma, \tau \in S_{q} .
$$

Since $\tau$ is bijective we can replace it by the identity. Setting

$$
\rho(\sigma)\left(\left(v_{1}, \ldots, v_{q}\right)\right)=\left(v_{\sigma^{-1}(1)}, \ldots, v_{\sigma^{-1}(q)}\right), \quad \sigma \in S_{q},
$$

defines an irreducible representation of $S_{q}$ on the space

$$
\left\{v \in \mathbf{C}^{q}: v_{1}+v_{2}+\cdots+v_{q}=0\right\}
$$

(see $[\mathbf{5}, \S 1.3]$ ). Therefore, if $C_{h l} \neq 0$ for some pair of indexes, a combination of vectors of the form $\left(C_{\sigma(1) j}, \ldots, C_{\sigma(q) j}\right)$ gives $\left(\overline{C_{h 1}}, \ldots, \overline{C_{h q}}\right)$. This contradicts (4.17).

Now, resuming the proof of Theorem 4.3, consider the set of automorphisms

$$
G_{S}:=\left\{g \in G: g\left(\left\{f_{1}, f_{2}, \ldots, f_{q}\right\}\right)=\left\{f_{q+1}, f_{2}, \ldots, f_{2 q}\right\}\right\} ;
$$

that is, with the notation of Definition 1 in Section 3 , the coset $j K^{\prime}$. When $g \in G_{S}, K_{\mathcal{I}} g$ is contained in $g K_{\mathcal{I}}$, so that $T^{g}$ is $K_{\mathcal{I}}$-invariant (since $T$ is). For $g \in G_{S}$ there exist $\sigma, \tau \in S_{q}$ such that

$$
\pi(g) f_{i}= \begin{cases}f_{q+\sigma(i)} & \text { if } i \leq q \\ f_{\tau(i-q)} & \text { if } i \geq q+1 .\end{cases}
$$

From the hypotheses on $T$ we see that $T^{g}$, as an operator on the span of $\left\{f_{1}, \ldots, f_{2 q}\right\}$, is represented by the block matrix

$$
A^{g}:=\left(\begin{array}{cc}
\lambda I_{q} & D^{\tau, \sigma} \\
C^{\sigma, \tau} & \lambda I_{q}
\end{array}\right)
$$

with $C^{\sigma, \tau}, D^{\tau, \sigma}$ defined as in Lemma 4.4. Hence

$$
A^{g} A=\left(\begin{array}{cc}
\lambda^{2} I_{q}+D D^{\tau, \sigma} & \lambda D^{\tau, \sigma}+\lambda C \\
\lambda D+\lambda C^{\sigma, \tau} & \lambda^{2} I_{q}+C C^{\sigma, \tau}
\end{array}\right) .
$$


Repeating the reasoning above for $T^{g}$ and $T^{g} T$ we find that $\widetilde{T^{g} T}$ is nonscalar for some $g \in G_{S}$, unless the blocks along the diagonal of $A^{g} A$ are scalar. Then $C C^{\sigma, \tau}$ and $D D^{\tau, \sigma}$ are also scalar, and since $\operatorname{det} C=\operatorname{det} D=0$ we get $C C^{\sigma, \tau}=D D^{\tau, \sigma}=0$ for every $\sigma, \tau \in S_{q}$. By Lemma 4.4, $C=D=0$, which is impossible because $T$ is nonscalar. This concludes the proof in case (ii).

Case (iii) is dealt with much like case (ii). Indeed, let $\left(x_{1}, x_{2}, \ldots, x_{n}\right)$ $(n \geq 3)$ be the central chain of the subtree:

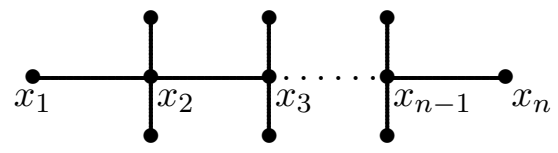

Figure 12. A subtree of the kind considered in case (iii).

and recall the notations set in Sections 4.2 and 4.3. If $P^{\prime}$ or $Q^{\prime}$ can be chosen in $\left\{x_{2}, \ldots, x_{n-1}\right\}$ then a boundary vertex $R$ with $P^{\prime} \neq R^{\prime} \neq Q^{\prime}$ is easily found (for example, if $Q^{\prime}=x_{1}$, it suffices to choose $R \in B\left(x_{n}, 1\right) \sim\left\{x_{n-1}\right\}$ ). So we restrict to the case in which $\left\{P^{\prime}, Q^{\prime}\right\}=\left\{x_{1}, x_{n}\right\}$ and $T\left(\mathcal{H}^{R}\right) \subseteq \mathcal{H}^{R}$ for $P \neq R \neq Q$. Now the subspace

$$
\bigoplus_{R \neq P, Q} \mathcal{H}^{R}
$$

is invariant under conjugation by $H_{x_{1}}, H_{x_{n}}$ (defined as in (4.2)) and also by the subgroup of inversions that switch $x_{1}$ with $x_{n}$. So $T$ can be represented by a diagonal block matrix, the two diagonal blocks corresponding to the orthogonal subspaces

$$
\bigoplus_{R \neq P, Q} \mathcal{H}^{R} \quad \text { and } \quad \mathcal{H}^{P^{\prime}} \oplus \mathcal{H}^{Q^{\prime}}
$$

Next, we consider the operators

$$
\widetilde{T}:=\int_{H_{P}} \pi(h) T \pi\left(h^{-1}\right) d h \quad \text { and } \quad \widetilde{T}^{\prime}:=\int_{H_{Q}} \pi(h) T \pi\left(h^{-1}\right) d h
$$

and apply the same reasoning as in point (ii) to the block along the diagonal which corresponds to $\mathcal{H}^{P^{\prime}} \oplus \mathcal{H}^{Q^{\prime}}$.

We deal finally with case (i). Let $O$ be the unique interior vertex of $\mathcal{I}$, and let $A$ be the $(q+1) \times(q+1)$ matrix of $T$ with respect to the basis $\left\{\mathbf{1}_{\Omega(O, P)}: P \in \partial \mathcal{I}\right\}$. Suppose that, for every $P \in \partial \mathcal{I}$,

$$
\widetilde{T}:=\int_{H_{O P}} \pi(h) T \pi\left(h^{-1}\right) d h
$$

$$
\text { where } H_{O P}:=\{h \in G: g O=O \text { and } h P=P\}
$$

is scalar. Then, as in case (ii), the following conditions on $A$ are found:

- In every row and column, nondiagonal entries add up to zero. 
- Every diagonal entry is the average of the $q$ remaining ones. Hence, all the diagonal entries are equal.

We can write $A$ as $\lambda I_{q+1}+A_{0}$, where $A_{0}$ satisfies the last two conditions and every diagonal element is zero. Now let $g$ be an automorphism that fixes $O$, and hence acts on $\partial \mathcal{I}$ as a permutation $\sigma$ on $q+1$ elements. The matrix of $T^{g}$ is $\lambda I_{q+1}+A_{0}^{\sigma, \sigma}$. Apply construction (4.26) to $T T^{g}$. If a scalar operator results for all $P \in \partial \mathcal{I}$, then $T T^{g}$ satisfies the same conditions as $T$. Consideration of the action of $T T^{g}$ on the vector $(1,1, \ldots, 1)$ shows that the common value of the diagonal elements is $\lambda^{2}$. It follows that $A_{0} A_{0}^{\sigma, \sigma}=0$ for every permutation $\sigma$. As in case (iii), Lemma 4.4 gives $A_{0}=0$, hence $A \in \mathbf{C} I_{q+1}$, a contradiction.

This completes the proof of Theorem 4.3 and hence of Theorem 4.1.

Acknowledgements. The results of this paper are contained in the author's doctoral thesis (Università di Roma "La Sapienza", 1998), written under the supervision of Professor Tim Steger. The author wishes to thank Professor Steger for many helpful conversations.

\section{References}

[1] P. Cartier, Géométrie et analyse sur les arbres, Sém. Bourbaki, Vol. 1971/1972, Exposés 400-417 and Lecture Notes in Math., 317, Springer, Berlin, 1973, 123-140, MR 0425032 (54 \#12990), Zbl 0267.14010.

[2] P. Cartier, Harmonic analysis on trees, Harmonic analysis on homogeneous spaces (Williamstown, MA, 1972), Proc. Sympos. Pure Math., 26, American Mathematical Society, Providence, 1973, 419-424, MR 0338272 (49 \#3038), Zbl 0309.22009.

[3] A. Figà-Talamanca and C. Nebbia, Harmonic Analysis and Representation Theory for Groups Acting on Homogeneous Trees, London Mathematical Society Lecture Note Series, 162, Cambridge University Press, Cambridge, 1991, MR 1152801 (93f:22004).

[4] A. Figà-Talamanca and T. Steger, Harmonic analysis for anisotropic random walks on homogeneous trees, Mem. Amer. Math. Soc., 110(531) (1994), MR 1219707 (95a:22003), Zbl 0836.43019.

[5] W. Fulton and J. Harris, Representation Theory: A First Course, Graduate Texts in Mathematics, 129, Springer, New York, 1991, MR 1153249 (93a:20069), Zbl 0744.22001.

[6] S.A. Gaal, Linear Analysis and Representation Theory, Grundlehren der mathematischen Wissenschaften, 198, Springer, Berlin, 1973, MR 0447465 (56 \#5777), Zbl 0275.43008 .

[7] A.W. Knapp, Representation Theory of Semisimple Groups: An Overview Based on Examples, Princeton Math. Ser., 36, Princeton University Press, Princeton, 1986, MR 0855239 (87j:22022), Zbl 0604.22001.

[8] G.W. Mackey, The Theory of Unitary Group Representations, Chicago University Press, Chicago-London, 1976, MR 0396826 (53 \#686), Zbl 0344.22002. 
[9] M.A. Nămark and A.I. Štern, Theory of Group Representations, Grundlehren der mathematischen Wissenschaften, 246, Springer, Berlin, 1982, MR 0793377 (86k:22001), Zbl 0484.22018.

[10] G.I. Ol'šanskiǔ, Classification of irreducible representations of groups of automorphisms of Bruhat-Tits trees, Funkcional. Anal. i Priložen., 11 (1977), 32-42 (in Russian; English translation, Functional Anal. Appl., 11 (1977), 26-34), MR 0578650 (58 \#28255), Zbl 0371.22014.

[11] G.K. Pedersen, Analysis Now, Graduate Texts in Mathematics, 118, Springer, New York, 1989, MR 0971256 (90f:46001), Zbl 0668.46002.

[12] T. Steger and M.K. Vemuri, Inductive algebras for $\operatorname{SL}(2, \mathbf{R})$, Illinois J. Math., to appear.

[13] M.K. Vemuri, Realizations of the canonical representation, preprint.

Received June 28, 2000 and revised October 22, 2003.

ISTITUTO DI MATEMATICA E FISICA

UNIVERSITÀ DI SASSARI

VIA ViEnNA 2, 07100 SASSARI

ITALY

E-mail address: stegel@uniss.it 